\title{
Ensino-Aprendizagem da Metodologia de Pesquisa Qualitativa em Medicina
}

\author{
Teaching-Learning of Qualitative Research \\ Methods in Medicine
}

Stella Regina Taquette Maria Cecília de Souza Minayo ${ }^{I I}$

\section{PALAVRAS-CHAVE}

- Pesquisa Qualitativa;

- Currículo Médico;

- Educação Médica;

- Conhecimento Científico;

- Humanização.

\section{KEYWORDS}

- Qualitative Research;

- Medical Curriculum;

- Medical Education;

- Scientific Knowledge;

- Humanization.

Recebido em: 17/08/2014

Aprovado em: 22/08/214
${ }^{1}$ Universidade Estadual do Rio de Janeiro, Rio de Janeiro, RJ, Brasil.

${ }^{\text {II }}$ Fundação Oswaldo Cruz, Rio de Janeiro, RJ, Brasil 


\section{NTRODUÇÃO}

O método qualitativo de pesquisa em saúde, compreendido como aquele que se ocupa com um nível de realidade que é tratado por meio da história, da biografia, das relações, do universo dos significados, dos motivos, das aspirações, das crenças, dos valores e das atitudes ${ }^{1}$, é de fundamental importância para dar respostas a problemas que se apresentam no dia a dia do médico em sua prática assistencial. Cassorla ${ }^{2}$ defende que trabalhar com o método qualitativo, que tenta compreender o significado dado pelo sujeito às suas ações, é estar próximo do que faz o médico clínico, que em seu ofício procura conhecer profundamente seu paciente, para além dos sinais e sintomas que apresenta. Esse autor destaca que os métodos qualitativos são semelhantes ao método da clínica, que, segundo os preceitos que a fundamentam, é soberana, e os demais exames são subsidiários.

No entanto, as pesquisas nas ciências da saúde, em sua maioria, são desenvolvidas com método quantitativo, lógico, experimental e matemático, com predileção pelo fenômeno extenso, que cultiva a objetividade e a neutralidade, hipotético-dedutivo, replicável e generalizável ${ }^{3}$. As que fogem a esta regra são realizadas geralmente por pesquisadores não médicos ou por aqueles que se debruçam mais sobre os problemas de saúde coletiva e não exercem diretamente a prática clínica. É bem verdade que a linguagem reflexiva das ciências sociais utilizada nos textos da área é pouco usual aos médicos, que têm formação eminentemente técnica. Alguns poucos profissionais de saúde que trabalham com o método qualitativo não raro são criticados pela superficialidade com que abordam a realidade social, pela incapacidade de debater os dados empíricos e por falhas na aplicação consistente e aprofundada da teoria ${ }^{4,5,6}$

Os médicos trabalham com seres humanos, cuja subjetividade se expressa no funcionamento do corpo, na saúde e na doença. Segundo Canguilhen ${ }^{7}$, a medicina é uma das ciências mais intimamente ligadas ao conjunto da cultura, já que qualquer transformação nas concepções médicas é condicionada pelas transformações ocorridas nas ideias da época. Por estes motivos, evidencia-se a importância de o médico ter no método qualitativo uma forma de abordagem indispensável ao conhecimento de questões relevantes que afetam seus pacientes do ponto de vista individual e coletivo.

Este artigo, que é parte de uma reflexão mais ampla baseada em pesquisa sobre a utilização do método qualitativo por médicos, foi desenvolvido com o objetivo de compreender a razão da pouca utilização do método por esses profissionais e conhecer a percepção destes sobre as possibilidades de ampliar seu uso no ensino, na pesquisa e na prática clínica.

\section{MATERIAL E MÉTODOS}

O público-alvo do estudo são médicos que, ao mesmo tempo, são pesquisadores e possuem doutorado. Optamos por esse grupo por entender que ele inclui os que têm maior experiência em pesquisa. Escolhemos o método qualitativo, dada a natureza subjetiva do objeto em questão. As informações foram colhidas em ambiente de intersubjetividade, por meio de entrevistas semiabertas. A entrevista é um instrumento privilegiado: ela nos permite conhecer,por meio da fala dos interlocutores,o sistema de valores de seu grupo social;é reveladora de suas condições estruturais;e, ao mesmo tempo, transmite as representações grupais, em condições históricas, socioeconômicas e culturais específicas ${ }^{1}$.A amostra foi intencional e composta por pesquisadores com prática de pesquisa tanto predominantemente quantitativa, quanto qualitativa. Participaram profissionais de diferentes instituições, programas de pós-graduação e especialidades médicas e com tempo de atividade variada na área de saúde. Para diversificar, ampliamos a amostra por vários Estados e, nesses casos, realizamos a entrevista por e-mail ou por skype.

Utilizamos um roteiro previamente testado com perguntas objetivas sobre dados demográficos, formação e experiência profissional, e questões semiabertas sobre barreiras na utilização do método e sugestões de como ensinar e ampliar seu uso pelos médicos. O convite para a participação no estudo foi feito pessoalmente ou por e-mail, com posterior agendamento da data e horário da entrevista. $\mathrm{O}$ critério de inclusão foi ter graduação em Medicina e ser pesquisador e doutor.

Três entrevistadoras participaram da coleta de dados, duas da área médica e uma de serviço social. Gravamos as entrevistas na íntegra, com consentimento dos entrevistados (com exceção das realizadas por e-mail), e as transcrevemos logo em seguida. Analisamos os dados à medida que foram coletados e consideramos a amostra completa e suficiente após a percepção clara de que seus conteúdos estavam amplamente saturados com base no conjunto das entrevistas. No total, entrevistamos 42 profissionais, sendo 18 com maior experiência no uso do método qualitativo e 24 no quantitativo. Trinta entrevistas orais consumiram 17 horas de gravação. E12 por e-mail resultaram na média de três páginas de texto.

A análise dos dados coletados compreendeu os seguintes passos: leitura e releitura dos textos, para termos uma visão singular de cada entrevista, e uma leitura transversal de todas, para compreensão dos conteúdos mais relevantes; classificação dos relatos e recorte e colagem do texto de acordo com categorias predefinidas: barreiras à utilização do método, ensino do método qualitativo e ampliação do uso do método por médicos; identificação dos sentidos atribuídos pelos sujeitos às 
questões levantadas, buscando entender a lógica interna deste grupo em diálogo comparativo com a literatura; elaboração de uma síntese interpretativa que respondesse aos questionamentos do estudo.

A pesquisa cumpre os princípios éticos contidos na Declaração de Helsinki, tendo sido aprovada pelo Comitê de Ética em Pesquisa do Hospital Universitário Pedro Ernesto em 12/12/12, parecer $\mathrm{n}^{\mathrm{o}}$ 155.405. Os participantes assinaram o Termo de Consentimento Livre e Esclarecido.

\section{RESULTADOS E DISCUSSÃO}

\section{Caracterização do grupo}

A maior parte dos participantes é formada por profissionais experientes, com tempo médio de doutoramento de 12,5 anos. Do total, 28,5\% são bolsistas de produtividade em pesquisa do CNPq. Todos são de instituições de ensino superior localizadas em três Estados: São Paulo (USP, UFSCar, Unifesp, Unicamp, Uam), Rio de Janeiro (Uerj, UFRJ, Fiocruz) e Minas Gerais (UFMG). Suas especialidades são variadas. Os que trabalham com estudos qualitativos são principalmente pesquisadores da área de Saúde Coletiva e Psiquiatria. Os que utilizam prioritariamente abordagens quantitativas são de especialidades clínicas e trabalham com Epidemiologia.

\section{Barreiras no uso do método}

A principal barreira apontada pela maioria dos entrevistados é a falta de preparação em ciências sociais. A formação médica é baseada nas ciências naturais, o que leva o médico a pensar e a agir usualmente segundo a lógica racionalista. Isto dificulta o aprendizado de temas subjetivos. Ou seja, de fato, o médico não desdenha a intersubjetividade, mas considera que ela não é um problema de sua alçada, eminentemente técnica. Esta é a percepção preponderante no grupo de entrevistados que utiliza predominantemente o método quantitativo em suas pesquisas. Houve quem dissesse que na graduação nunca "foi apresentado ao método", nem ouviu falar que existia outro tipo de investigação que não fosse quantitativa. Canesqui ${ }^{6}$, em suas reflexões sobre a presença das ciências sociais e humanas na Saúde Pública, argumenta que "pode-se dizer que a medicina continua ensurdecida à pessoa humana" (p. 18) ao comentar a dificuldade de o profissional dessa área, em sua prática, abandonar a postura de centralidade no indivíduo, sem levar em consideração o contexto social.

Geralmente, os pesquisadores que trabalham com abordagens estatísticas consideram científico somente o estudo cujos resultados sejam replicáveis e generalizáveis. Suas narrativas demonstram a percepção de que apenas as pesquisas quantitativas são científicas:

\begin{abstract}
"Prevalece no mundo científico a mais valia das áreas quantitativas, mensuráveis, desde Galileu".

"Eu sou muito quantitativo, a começar pela minha maneira de pensar, eu penso de forma muito quantitativa, muito estruturada. Eu tenho uma mente probabilística, então eu acho que isso já é um empecilho para pensar de forma qualitativa". "Não há muito conhecimento e então acaba havendo muita resistência. Quando isso acontece, se fecha muito o pensamento do pesquisador".
\end{abstract}

Tanto para os pesquisadores que trabalham com abordagens quantitativas como para os que utilizam as qualitativas, a falta de formação é a principal barreira para o uso dos métodos compreensivos, uma vez que eles exigem competências específicas, como ter fundamentos filosóficos e sociológicos, e saber compreender e interpretar. Tais habilidades dependem de aprendizado em ciências humanas e sociais, o que, em geral, o médico não tem.

Outro aspecto assinalado é a postura positivista fechada, que leva a uma simplificação exagerada do que é dito pelos entrevistados, pois nas abordagens quantitativas não cabe o processo interpretativo: apenas o que é dito e escrito pode ser considerado. De forma geral, é o desconhecimento do método qualitativo pelos médicos o ponto que mais explica a rejeição e desvalorização:

\begin{abstract}
"A gente não é treinado para isso, a gente não tem preparação nenhuma para pesquisa no currículo da faculdade. Por conta disso, as pessoas ficam descrentes ou não apostam em sua capacidade de produzir conhecimento desta forma".

"A gente não aprende a ler e não tem nenhuma formação humanista. Você tem que ter um olhar que tudo tem história, tudo tem correlações de forças, tudo tem valores culturais que concorrem, competem, compõem ou negociam, quer dizer, nada vem do nada".
\end{abstract}

Foram apontadas também como barreiras: as questões de subjetividade que favorecem a ocorrência de vieses, como, por exemplo, a dificuldade do pesquisador em se distanciar do que investiga e o risco de misturar sua opinião com os dados que recolhe no campo; o tempo que uma pesquisa de campo exige; as dificuldades para analisar os dados; e os problemas que os pesquisadores qualitativos enfrentam para publicar seus artigos.

O envolvimento do pesquisador com o pesquisado é uma das características da abordagem qualitativa. Ressaltamos que fazer pesquisa qualitativa, principalmente na área da saúde, significa ser solidário e compartilhar transformações a favor 
dos grupos estudados ${ }^{8}$. Assim como faz parte da ética dessa abordagem levar em conta a influência que a relação pesquisador/pesquisado tem sobre os resultados da pesquisa. Os critérios para avaliar a qualidade de um estudo interagem com as normas éticas da investigação, como é o caso da transparência sobre como foi realizado o trabalho de campo e como se conseguiram as informações, como foi feita a análise e apresentação dos dados, inclusive para deixar claras as relações de poder entre pesquisador e pesquisado ${ }^{9}$.

A dificuldade de publicação de artigos científicos que usam abordagem qualitativa na área da saúde, apontada pelos entrevistados, encontra ressonância na literatura médica mundial. Um estudo de revisão sobre pesquisas publicadas nas 170 principais revistas clínicas no ano de 2000 revelou que, de 60.330 artigos revistos, apenas $355(0,5 \%)$ eram de estudos qualitativos, publicados em 48 diferentes periódicos, sendo a maioria da área de Enfermagem e somente quatro em revistas de alto fator de impacto ${ }^{10}$. Outro estudo, também de revisão de artigos sobre serviços de saúde e de gestão, publicados nos nove principais periódicos dessas áreas num período de três anos (1995-97), mostra que apenas um em cada sete artigos utilizou métodos qualitativos ${ }^{11}$.

De acordo com Morse ${ }^{12}$, editora da revista Qualitative Health Research, a investigação qualitativa continua à margem da ciência, apesar dos esforços para garantir seu rigor. Na opinião da autora, médicos teimam em considerar as abordagens qualitativas muito subjetivas, tendenciosas, não replicáveis, sem apresentação de evidências e, portanto, inaceitáveis. Embora esses profissionais, teoricamente,tenham na metodologia qualitativa um campo de conhecimento privilegiado, pois suas experiências na assistência permitem a realização de importantes estudos de base compreensiva, poucos a utilizam. Igualmente, os poucos artigos que os médicos escrevem e que utilizam essa abordagem costumam ser rejeitados por pareceristas, que os consideram não científicos, de qualidade duvidosa, como se fossem apenas histórias curiosas sobre eventos vividos,que pouco avançam além do senso comum ${ }^{13}$.

\footnotetext{
"Existe certa desqualificação oficial do método (qualitativo) por parte das principais revistas científicas da área médica". "É um método artesanal, que demanda bastante treinamento por um tempo relativamente longo. Exige capacitação prévia em ciências sociais".
}

Apesar das dificuldades, o número de publicações de estudos qualitativos está crescendo, assim como sua aceitação pelos periódicos. Segundo revisão de Yamazaki ${ }^{14}$ sobre artigos publicados entre 2000 e 2004 nas revistas de medicina geral de maior fator de impacto no mundo - BMJ, Lancet, Jama, Annals of Internal Medicine e New England Journal of Medicine -, $11 \%$ eram de estudos qualitativos. Em dez anos, as publicações qualitativas internacionais aumentaram 3,4 vezes $(1,2 \%$ em 1998 e 4,1\% em 2007) segundo Shuval ${ }^{15}$.

A evidência demonstrada pelos dados, no entanto, é de que o incremento nas publicações é ainda muito lento, e poucos periódicos importantes aceitam artigos dessa natureza. Aí se somam três barreiras assinaladas pelos entrevistados: a lógica do mundo acadêmico, que exige grande quantidade de artigos; que sejam publicados em revistas com alto fator de impacto, que, por sua vez, costumam não aceitar estudos com essa abordagem; e os claros obstáculos criados pelos comitês de ética científica, que quase nunca entendem projetos dessa natureza. Essa incompreensão tem um efeito colateral grave: alguns pesquisadores qualitativos imitam a lógica e a linguagem da ciência positivista para terem seus trabalhos aprovados e publicados.

\begin{abstract}
"Eu estou assustado de ver como pessoas que trabalham com a técnica qualitativa estão começando a imitar o pessoal da quantitativa. [...] Muitos fazem assim, [...] Como pode um artigo baseado em história de vida com seis autores? As pessoas estão querendo fazer número de artigos muito rápido. É uma busca de produção desenfreada que cria uma série de incentivos perversos. Isso na verdade é inimigo da ciência".
\end{abstract}

\section{Ensino do método qualitativo}

Os entrevistados ressaltaram a necessidade de iniciar o ensino do método qualitativo já na graduação, assim como é feito com o método quantitativo. Ao terem contato com boas pesquisas qualitativas, os alunos aprenderiam a importância delas. Isto promoveria uma redução do preconceito de considerar que as pesquisas qualitativas são desenvolvidas por pesquisadores menos competentes.

Ainda segundo os entrevistados, uma forma importante de ensinar pesquisa qualitativa é orientar o estudante a refletir sobre questões da sua prática profissional, levando-o a formular perguntas de pesquisa - por exemplo, sobre como os pacientes tomam decisões sobre seu tratamento, sobre a forma como utilizam os medicamentos. Discussões e pesquisas como essa são fundamentais para a formação, pois existe uma tendência dos médicos a evitar o diálogo, a compreensão dos problemas e ir direto ao ponto técnico em que querem atuar. Então, como já nos lembrava Boltanski em 1979¹6, saúde e doença são sempre mediadas pela cultura, ou seja, por concepções, crenças, representações e valores. 
"Na verdade, na pesquisa qualitativa a gente não vê tanto peso. Então, uma maneira de estimular seria ser apresentado a ela. No início (de uma disciplina qualitativa que fez na $P G)$, eu achei a maior viagem, mas depois percebi que era um negócio legal".

Os pesquisadores entrevistados consideram que o principal elemento para o ensino do método e, consequentemente, para ampliação do seu uso é a comprovação prática de sua utilidade. Outra sugestão importante para o ensino é fazê-lo de modo concreto, ou seja, ensinar fazendo pesquisa, utilizando técnicas novas que estimulem os estudantes nos diversos ambientes em que isto seja possível. Esta também é a opinião de diferentes pesquisadores, veiculada em debate no Reserachgate $^{17} \mathrm{em}$ resposta à pergunta "What is the best way to teach qualitative research?".A maioria destacou que a melhor forma de aprender é fazendo.

A inclusão de disciplinas de humanidades e sociais na graduação e na pós-graduação foi apontada por quase todos os entrevistados como essencial para aumentar o conhecimento dos métodos e os conceitos estruturantes da investigação qualitativa. A compreensão e a interpretação dos dados empíricos coletados na pesquisa de campo permitem a elaboração científica, o construto de segunda ordem, ou seja, chegar à compreensão da lógica que preside a fala dos entrevistados e conectá-la com a literatura universal ${ }^{18,19}$.

Nesse debate do Researchgate ${ }^{17}$, alguns pesquisadores afirmam que a maior dificuldade encontrada é fazer os alunos entenderem o que é um quadro teórico e sua importância, sempre tendo em conta que a realidade social pode ser compreendida e interpretada através da lente de várias teorias. Acreditamos, porém, que essa dificuldade deve ser decomposta: tendo em vista que a medicina trabalha com marcos explícitos ou implícitos da ciência positivista, o estudante precisa ser exposto a uma reflexão sobre os fundamentos teóricos consagrados da Antropologia e da Sociologia compreensiva, segundo os quais podemos trabalhar cientificamente com o senso comum e com a subjetividade e daí criar conhecimentos úteis para a prática médica. Entenda-se aqui que o senso comum e qualquer outra manifestação de subjetividade são matéria-prima para compreensão e interpretação.

Alguns autores argumentam que o conhecimento da teoria social é tão importante para a pesquisa qualitativa de alta qualidade como o conhecimento da estatística na condução dos estudos epidemiológicos. Ele pode gerarimportantes insights sobre a experiência social. Na verdade, muitos trabalhos realizados com primor permitem extrapolar as conclusões mi- crossociais para configurações e grupos mais amplos do que aqueles em que a pesquisa focalizada foi conduzida ${ }^{20}$.

Há certa unanimidade em algumas falas dos entrevistados, tanto da linha quantitativa como da qualitativa, quanto à necessidade de melhorar a formação humanista do médico. Todos concordam também em que, em geral, há falta de interesse dos estudantes por metodologia do ponto de vista teórico. Por isso, a abordagem e as técnicas devem ser ensinadas na prática, na participação em projetos de pesquisa e com a apresentação de exemplos simples que possam ser aproveitados na atenção médica. Alguns entrevistados consideram que o aumento do conteúdo humanista na formação médica contribuirá para que tenhamos melhores médicos.

\begin{abstract}
"Eu acho que devia se trabalhar um pouco a história do pensamento científico. Tem que sair do estado de inocência. O cara pode ser um excelente pesquisador numa área médica disso e daquilo, mas ele tem uma ingenuidade histórica".
\end{abstract}

De acordo com Turato $^{13}$, a proximidade com o campo e com os próprios sujeitos de pesquisa na área de saúde coloca os médicos numa posição vantajosa em relação aos problemas de pesquisa de natureza qualitativa por causa de sua experiência na assistência, no contato com as pessoas e na compreensão de seus problemas. No entanto, essa aproximação não é dada. Ela é construída e mediada por estratégias de aproximação, de entendimento e de interpretação $0^{1,21,22}$. Chase ${ }^{23}$ defende que a melhor maneira de ensinar os alunos a realizar pesquisas qualitativas com base em entrevistas em profundidade é usar os princípios dos estudos de narrativa. $\mathrm{O}$ autor mostra como esses princípios incentivam os alunos a ouvir com atenção seus interlocutores e, consequentemente, melhoram sua prática de investigação, em especial na elaboração de perguntas e na realização de entrevistas.

Reflexões a respeito da necessidade de utilizar o método qualitativo em saúde para abordar os problemas que se apresentam na prática médica deram origem a uma proposta de ensino-aprendizagem desenvolvida por Calderón ${ }^{24}$, médico de família de um Centro de Atenção Primária de San Sebastian, na Espanha. Calderón destaca que o trabalho assistencial tem uma complexidade para a qual é necessário um olhar qualitativo quando se pretende responder a questões que incluem valores, crenças, representações, atitudes e comportamentos sobre a saúde e a doença. Para isso, é preciso haver mão dupla na articulação das ciências sociais e humanas com a medicina. Os cientistas sociais têm que saber traduzir a linguagem e os métodos com os quais trabalham, tornando-os acessíveis aos profissionais de saúde ${ }^{25}$. 
Weiner ${ }^{26}$ sugere que o ensino de metodologia qualitativa seja obrigatório nos programas de doutorado, mesmo para aqueles não pretendem utilizar essa abordagem. Isto porque tal aprendizagem pode proporcionar maior valorização das investigações empíricas e maior habilidade para avaliação da qualidade científica dos estudos, uma vez que esses alunos se tornarão revisores e editores de revistas.

\section{Ampliar e tornar mais acessível o uso do método qualitativo por médicos}

As narrativas dos pesquisadores quantitativistas e qualitativistas foram semelhantes neste particular. Para ampliar o uso do método qualitativo, elas destacam a necessidade de torná-lo mais acessível na linguagem e mediante exemplos, e útil para a prática médica. Alguns médicos que o conhecem pouco o abordam, o rejeitam ${ }^{27}$ e até consideram impossível trabalhar com ele, pois já têm "a cabeça formatada" para o quantitativo. Acontece que, na maioria dos casos, os termos e conceitos utilizados nos textos teóricos sobre metodologia de pesquisa qualitativa não são atraentes para médicos, que, muitas vezes, não conseguem compreender sobre o que se está falando, tal o distanciamento entre sua prática e essa proposta.

Segundo a lógica racional em que são formados os médicos, é preciso mostrar para que serve o conhecimento qualitativo. Alguns se referiram à necessidade de, na docência, tornar as aulas agradáveis e mostrar possíveis soluções de problemas do dia a dia sob a perspectiva compreensiva. Um dos entrevistados lembrou que, ao contrário dos pesquisadores médicos, empresas comerciais gastam fortunas com pesquisas qualitativas para entender os interesses de seus consumidores.

\footnotetext{
"Se nós pegássemos questões em que ficasse bem evidente a importância de você ter o estudo qualitativo, como a não aderência de um paciente ao tratamento, por exemplo".
}

Apesar da pouca credibilidade dos métodos qualitativos no meio médico, há uma preocupação crescente, segundo alguns autores ${ }^{28}$, de que a medicina baseada em evidência não tem influenciado suficientemente o desenvolvimento da prática profissional, criando espaço, sobretudo, para a pesquisa-ação. Esta é uma abordagem cujas características são a investigação sobre a prática com instrumentos que focalizam o processo e os resultados enquanto o estudo está acontecendo.

Segundo os entrevistados, a qualificação dos pesquisadores pode ser melhorada por meio de cursos de metodologia que desmistifiquem a dicotomia existente entre as abordagens quantitativas e qualitativas, buscando uma integração desses estudos, cujos exemplos já são bastante comuns ${ }^{29}$. Soma-se a isso a leitura de bons livros-textos sobre o tema, que diferencie um objeto de pesquisa qualitativa da quantitativa, de autores seminais, como Denzin ${ }^{30}$, por exemplo.

$\mathrm{Cano}^{31}$ defende a ideia de que, a rigor, a separação entre método quantitativo e método qualitativo é artificial. A quantificação ou não das mensurações é apenas o aspecto exterior e visível, mas secundário, de qualquer estudo. Os dilemas epistemológicos enfrentados pelo etnógrafo ou pelo profissional que trabalha com surveys são os mesmos. No entanto, numa contraposição ao que propõe Cano, lembramos que os pesquisadores que usam métodos qualitativos não costumam se questionar sobre a cientificidade de seus processos de construção científica, embora eles existam, uma vez que toda evidência científica, obtida seja por método quantitativo,seja por qualitativo, tem que ser avaliada com cuidado para poder oferecer conclusões válidas. As pesquisas quantitativas também dependem do contexto para interpretar seus resultados.

Para ampliar e qualificar o conhecimento dos pesquisadores médicos, alguns entrevistados sugeriram incorporar investigadores competentes no uso de método qualitativo aos grupos de pesquisa clínica, assim como incentivar a realização de trabalhos interdisciplinares entre áreas de ciências sociais e humanas e áreas médicas, com o cuidado de incluir jovens pesquisadores. Os entrevistados ressaltaram que os pesquisadores que trabalham com o método qualitativo precisam se empenhar mais em divulgar seus estudos em congressos e na instituição em que atuam. Muitos sublinharam a quase inexistência deste tipo de informação dentro da universidade:

“O método qualitativo ninguém sabe, ninguém aprende. Não tem essa divulgação, as pessoas não conhecem quem não está envolvido nessa área não conhece".

A divulgação mais efetiva da importância do método qualitativo e suas potencialidades entre docentes das faculdades de Medicina é uma estratégia relevante. Os profissionais médicos que têm experiência e competência com o método qualitativo precisam ser convocados para partilhar com seus pares sua experiência, ampliando o conhecimento dos colegas sobre o tema.

\section{ALGUMAS CONSIDERAÇÕES}

O uso do método qualitativo de pesquisa por médicos depende fortemente de investimento na formação desses profissionais desde a graduação. Nos programas de pós-graduação, é importante incluir a metodologia qualitativa como disciplina obrigatória, mesmo para aqueles que pesquisam problemas que não necessitem de um olhar qualitativo. Todos os pós- 
-graduandos devem conhecer o método, que, na maioria dos casos, faz uma articulação enriquecedora com o quantitativo. Esse investimento na formação amplia o conhecimento metodológico dos estudantes, tornando-os competentes para desenvolver investigações que abordem questões subjetivas e relacionais relevantes para a saúde. Pelo menos, esse conhecimento lhes ensinará a compreender e a respeitar uma metodologia alternativa à que usam em sua prática.

Por outro lado, o ensino do método qualitativo também é motivo de preocupação, uma vez que, frequentemente, muitos cientistas sociais encontram dificuldade na docência. A literatura sobre ensino de metodologia qualitativa é escassa, e os pesquisadores qualitativos ocupam posição marginal em algumas instituições. Não existe nas ciências sociais um modelo de método qualitativo para gerar conhecimentos que seja uniforme, e nem isso deveria ocorrer, tendo em vista a pluralidade de propostas existentes na atualidade. Segundo Breu$\mathrm{er}^{32}$, os modelos de pesquisa qualitativas e alternam em dois polos extremos, sendo um marcado por uma concepção paradigmática e holística, e o outro, por práticas metodológicas pragmáticas. Esse autor destaca também a grande importância da relação pessoal entre professores e alunos no contexto educacional, pois a pesquisa qualitativa envolve proximidade entre os protagonistas e mais reflexão interpessoal. $\mathrm{O}$ mesmo mostrou Minayo ${ }^{33}$ num ensaio crítico sobre o ensino das ciências sociais em saúde. Nesse estudo, a autora mostra que, na atualidade, o campo se divide entre os que creem que as ciências sociais deveriam produzir crítica sobre os problemas, e os que buscam produzir informações que possam ser apropriadas pela sociedade.

Para alguns, existem pré-requisitos para se engajar em pesquisa qualitativa, como, por exemplo, estar aberto e disposto a refletir sobre si mesmo e suas ações; ter capacidade de compreender, de ter uma atuação em intersubjetividade com os entrevistados, de interpretar os dados primários à luz de uma consistente visão teórica; ter competência para lidar com conflitos, ser criativo, paciente e persistente. Outros argumentam não terem dúvidas quanto à influência de fatores biográficos dos autores e também dos alunos na escolha do método quando planejam suas pesquisas ${ }^{34}$. Na verdade, temos pouco conhecimento sobre ensino do método qualitativo. Ao longo do tempo, houve negligência no treinamento dos educadores porque, entre outros motivos, as pesquisas dessa natureza eram consideradas uma forma de arte e não um conjunto de procedimentos sistemáticos e científicos ${ }^{35}$.

Para uma compreensão mais ampla das carências da formação médica que resultam no desconhecimento ou desvalorização do método qualitativo de pesquisa, é necessária uma avaliação cuidadosa dos currículos das escolas médicas no que diz respeito ao conteúdo de ciências sociais e humanas. Essa avaliação não foi feita em nosso estudo, o que pode limitar a abrangência de seus resultados. Entretanto, as informações aqui contidas, a nosso juízo, servem para acelerar um debate fundamental na formação dos estudantes de Medicina.

\section{AGRADECIMENTOS}

As autoras agradecem aos colegas médicos, sujeitos de pesquisa deste estudo; à bolsista de capacitação técnica da Faperj Adriana de Oliveira Rodrigues; à bolsista de iniciação científica da Faperj Antonia Ivoneide Correia Viana; e à Faperj, pelo apoio financeiro concedido.

\section{REFERÊNCIAS}

1. Minayo MCS. O desafio do conhecimento. Pesquisa qualitativa em saúde. 13. ed. São Paulo: Hucitec; 2013. 407p.

2. Cassorla RMS. Prefácio. In: Turato ER. Tratado da Metodologia da Pesquisa Clínico-Qualitativa: construção teórico-epistemológica, discussão comparada e aplicação nas áreas da saúde e humanas. Petrópolis-RJ: Vozes; 2003. p. 19-32.

3. Demo P. Ciências sociais e quantidade. In: Demo P. Ciências sociais e qualidade. São Paulo, Almed, 1985.

4. Gomes MHA, Silveira C. Sobre o uso de métodos qualitativos em Saúde Coletiva, ou a falta que faz uma teoria. Rev Saúde Pública. 2012;46(1):160-5.

5. Carta ao Editor. Sobre fazer ciência na pesquisa qualitativa: um exercício avaliativo. Rev Saúde Pública. 2012;46(2):392394.

6. Canesqui AM. Sobre a presença das ciências sociais e humanas na saúde pública. Saúde Soc. 2011;20(1):16-21.

7. Canguilhen G. O normal e o patológico. 2.ed. Rio de Janeiro: Forense Universitário;1982. 270p.

8. Minayo MCS, Guerriero ICZ. Reflexividade como éthos da pesquisa qualitativa. Cienc Saúde Coletiva. 2014;19(4):1103-1112.

9. Fossey E, Harvey C, McDermott F, Davidson L. Unerstanding and evaluatina qualitative research. Australian and New Zealand Jornal of Psychiatry. 2002;36:717-732.

10. McKibbon K, Gadd CS. A quantitative analysis of qualitative studies in clinical journals for the 2000 year. BMC Medical Informatics and Decision Making. 2004;4(11).

11. Hoff TJ, Witt LC. Exploring the use of qualitative methods in published health services and management research. Medical Care Research and Review. 2000; 57:139-160.

12. Morse JM. Reconceptualizing Qualitative Evidence. Qual Health Res. 2006;16(3):415-422. 
13. Turato ER. Métodos qualitativos e quantitativos na área da saúde: definições, diferenças, e seus objetos de pesquisa. Rev Saúde Pública. 2005;39(3):507-14.

14. Yamazaki H, Slingsby BT, Takahashi M, Hayashi Y, Sugimori H, Nakayama T. Characteristics of qualitative studies in influential journals of general medicine: a critical review. BioScience Trends. 2009;3(6):202-209.

15. Shuval K, Harker K, Roudsari B, Groce EM, Mills B, Siddiqi $\mathrm{Z}$ and Shachak A. Is Qualitative Research Second Class Science? A Quantitative Longitudinal Examination of Qualitative Research in Medical Journals. PLoS One. 2011;6(2) e 16937.

16. Boltanski, L. As classes sociais e o corpo. Rio de Janeiro: Editora Graal, 1979.

17. Dsane S. What is the best way to teach qualitative research? ).[acessado 10 jul.2014]. Disponível em: https:/ / www. researchgate.net/post/What_is_the_best_way_to_teach_ qualitative_research?pli=1\&loginT=koo67uSEmLmFM9L7aVa0YIJPGp7v0BdZ7wUSy4OqveTjW13UgtTnkA**\&uid $=9 e 6 c 8 f 0 e-8 c b 3-4654-a 6 b 9-5 f 50 a 29$ adeec\&cp =re221_sk_a_ ey_p42\&ch=reg.

18. Minayo MCS. Los conceptos estructurantes de la investigación cualitativa. Salud Coletiva. 2010;6(3):2-9.

19. Minayo MCS. Análise qualitativa: teoria, passos e fidedignidade. Cienc Saúde Coletiva. 2012;17(3):621-26.

20. 1. Willis K, Daly J, Kealy M, Small R, Koutroulis G, Green J, Gibbs L, Thomas S. The essential role of social theory in qualitative public health research. Aust N Z J Public Health.2007;31(5):438-43.

21. Cohn A. Ciências Sociais e Saúde Pública/Coletiva: a produção do conhecimento na sua interface. Saúde Soc. 2013;22(1):15-20.

22. 2. Baxter S, Killoran A, Kelly MP, Goyder E. Synthesizing diverse evidence: the use of primary qualitative data analysis methods and logic models in public health reviews. Public Health.2010;124(2):99-106.

23. Chase, Susan E. "Learning to listen: Narrative principles in a qualitative research methods course." (2003).[acessado 10 jul.2014] Disponível em: https:/ / www.researchgate.net/publication/232445327_Learning_to_listen_Narrative_principles_in_a_qualitative_research_methods_ course.

24. Calderón C. La enseñanza-aprendizaje de la investigación cualitativa en el medio sanitario. Cienc Saúde Coletiva. 2012;17(3):595-602.

25. Marsiglia EMG. Temas emergentes em ciências sociais e saúde pública/coletiva: a produção do conhecimento na sua interface. Saúde Soc. 2013;22(1):32-43
26. 3.Weiner BJ, Amick HR, Lund JL, Lee SY, Hoff TJ. Use of qualitative methods in published health services and management research: a 10-year review. Med Care Res Rev.2011;68(1):3-33.

27. Santos SR. Métodos qualitativos e quantitativos na pesquisa biomédica. J Pediatr. 1999. p.401-406.

28. Meyer J. Qualitative research in health care. Using qualitative methods in health related action research. BMJ. 2000;320:178-81.

29. Minayo MCS, Assis SG. Avaliação por triangulação de métodos. Rio de Janeiro: Editora Fiocruz, 2007.

30. Denzin NK, Lincoln YS. Introdução. A disciplina e a prática da pesquisa qualitativa. In: Denzin NK, Lincoln YS (org). O Planejamento da Pesquisa Qualitativa. Teorias e Abordagens. 2a ed. Porto Alegre: Artmed; 2010. p.15-42.

31. Cano I. Nas trincheiras do método: ensino da metodologia das ciências sociais no Brasil. Sociologias. 2012;14(31):94-119.

32. Breuer F, Schreier M. Issues in learning about and teaching qualitative research methods and methodology in the social sciences." Forum Qualitative Sozialforschung/Forum: Qualitative Social Research. 2007; (8)1[acessado 10 jul.2014] Disponível em: http://www.qualitative-research.net/index.php/fqs/article/view/216/478.

33. Minayo MCS. Herança e promessas do ensino das Ciências Sociais na área da Saúde. Cad Saúde Pública. 2012;28(12):2367-2372.

34. Silverman D. What you can (and can't) do with qualitative research. ).[acessado 10 jul.2014]. Disponível em: http: / / www.uk.sagepub.com/upm-data /29042_02_ Silverman_3e_Ch_02.pdf .

35. Hurworth R. Theory, practice or both?: Views of experts. Qualitative Research Journal. 2004; 4(1):61.

\section{CONTRIBUIÇÃO DOS AUTORES}

Stella Regina Taquette e Maria Cecília de Souza Minayo participaram da concepção e desenho do estudo, análise e interpretação dos dados e redação do manuscrito.

\section{CONFLITO DE INTERESSES}

Declarou não haver.

\section{ENDEREÇO PARA CORRESPONDÊNCIA}

Stella Regina Taquette

Rua Gomes Carneiro, 34, apto 802

Ipanema - Rio de Janeiro

CEP: 22071-110 RJ

E-mail: stella.taquette@gmail.com 